\title{
Glucose-dependent insulinotropic polypeptide and insulin-like immunoreactivity in saliva following sham-fed and swallowed meals
}

\author{
B Messenger, M N Clifford and L M Morgan \\ School of Biomedical and Life Sciences, University of Surrey, Guildford, Surrey GU2 7XH, UK \\ (Requests for offprints should be addressed to L M Morgan; Email: I.morgan@surrey.ac.uk)
}

\begin{abstract}
Gastrointestinal peptides, including insulin, glucagon and glucose-dependent insulinotropic polypeptide (GIP) have previously been reported in salivary glands. Recent evidence has suggested they might influence postprandial macronutrient metabolism. This study therefore investigated and compared postprandial hormone concentrations in saliva and plasma to determine whether their secretion was influenced by oral food stimuli. In a within-subject randomised cross-over comparison of hormone concentrations in plasma and saliva following a mixed meal, 12 subjects were given two $1708 \mathrm{~kJ}$ mixed meals. On one occasion the meal was chewed and swallowed (swallowed meal), on the other it was chewed and expectorated (sham-fed meal). Salivary and plasma levels of immunoreactive insulin, GIP and glucagon-like peptide-1 (GLP-1), total protein, $\alpha$-amylase, glucose and nonesterified fatty acid were measured before and for $90 \mathrm{~min}$ following the meals. Saliva total protein and $\alpha$-amylase rose following both meals, indicating that the stimulus for salivary protein release is related to the presence of food in the mouth. GLP-1 was not detected in saliva. Fasting
\end{abstract}

salivary insulin levels were lower in saliva than plasma ( $28 \pm 6$ vs $40 \pm 25 \mathrm{pmol} / 1$ respectively). Both increased following the swallowed meal but the rise in saliva was slower and less marked than in plasma (peak levels $96 \pm 18$ and $270 \pm 66 \mathrm{pmol} / 1$ for saliva and plasma respectively, $P<0 \cdot 01)$. Both were unchanged following the sham-fed meal. GIP was detected in saliva. Fasting GIP levels were significantly higher in saliva than plasma $(183 \pm 23$ compared with $20 \pm 7 \mathrm{pmol} / 1, P<0 \cdot 01)$. They decreased in saliva following both swallowed and sham-fed meals to nadirs of $117 \pm 17$ and $71 \pm 12 \mathrm{pmol} / 1$ respectively, but rose following the swallowed meal to peak levels of $268 \pm 66 \mathrm{pmol} / 1$. These findings are consistent with insulin in saliva being an ultrafiltrate of that circulating in blood, but GIP in saliva being the product of local salivary gland synthesis, whose secretion is influenced, directly or indirectly, by oral stimuli. The function of salivary GIP is unknown, but we speculate that it may play a role in the regulation of gastric acid secretion in the fasting state.

Journal of Endocrinology (2003) 177, 407-412

\section{Introduction}

Most mammals possess three pairs of salivary glands, the submandibular, parotid and sublingual glands, the secretory end-pieces of which are drained by a branched duct system to secrete saliva into the mouth. Saliva performs several functions, including lubricating the mouth to facilitate the swallowing of food and prevent the demineralisation of tooth enamel. $\alpha$-Amylase, secreted into saliva, catalyses the hydrolysis of ingested starch in the buccal cavity. The salivary glands are a rich source of biologically active peptides. A number of growth factors are synthesised within the salivary gland and secreted into saliva, including epidermal growth factor (Boyer et al. 1991), insulin-like growth factor-I and tumour necrosis factor- $\alpha$ (Humphreysbeher et al. 1994, Kerr et al. 1995).

The salivary glands also contain biologically active proteins involved in glucose homeostasis. Human saliva contains a proline-rich salivary peptide $\mathrm{P}-\mathrm{C}$, which potentiates glucose-induced insulin release and inhibits arginine-induced glucagon release in the rat pancreas (Kimura et al. 1995, Kimura et al. 1998). Insulin-like immunoreactivity has been extensively reported in rodent salivary glands, human parotid (Murakami et al. 1982) and submandibular salivary glands (Shubnikova et al. 1984), and in human saliva (Fekete et al. 1993). Glucagon-like immunoreactivity has been reported in rat salivary glands (Lawrence et al. 1977). A native form of peptidylglycine alpha-amidating monooxygenase is secreted into saliva (Kato et al. 1992); this is a regulating enzyme in the synthesis of biologically active hormones, such as glucagon-like peptide-1 (GLP-1), that have a C-terminal amide. The gastrointestinal (GI) hormone glucosedependent insulinotropic polypeptide (GIP) has also been detected in rodent salivary glands and saliva (Tseng et al. 1993, 1995). The transcripts have been detected in the 
striated ductal cells of the submandibular salivary gland. Pretranslational modulation of the GIP gene by nutrient ingestion has also been demonstrated in salivary glands as well as in the GI tract (Tseng et al. 1994, 1995), indicating that the GIP transcript is processed in the salivary gland to produce native GIP, consistent with a physiological role for salivary GIP in the rat.

The physical presence of food in the mouth may stimulate hormone secretion from the salivary glands, and salivary hormone secretion into saliva may be modulated by the physical action of masticating food in the mouth, rather than the presence in, or absorption of, food from the gut. Sham-feeding a high-fat test 'meal' following ingestion of encapsulated oil results in higher circulating triacyglycerol levels compared with sham-feeding a lowfat test 'meal' (Mattes 1996), suggesting that oral exposure to dietary fat can influence postprandial lipid metabolism. Sham-feeding during an intra-gastric glucose infusion has also been found to influence circulating plasma glucose levels (Teff \& Engleman 1996), and salivary peptide P-C has been found to modulate both insulin and glucagon release from the isolated pancreas (Kimura et al. 1990). These experiments indicate that the presence of food in the mouth has the ability to modulate both carbohydrate and lipid metabolism. These effects, consistent with the known biological actions of hormones such as insulin and GIP, might be mediated via the production of these hormones from the salivary glands.

This study was therefore designed to investigate and compare postprandial hormone concentrations in saliva and plasma following ingestion of a mixed meal, and to determine whether their secretion can be stimulated by the physical presence of food in the mouth, following a sham-fed meal, which was chewed and expectorated.

\section{Materials and Methods}

\section{Subjects}

Twelve healthy subjects, six men and six women aged 21-35 years with body mass indices ranging from 21 to $28 \mathrm{~kg} / \mathrm{m}^{2}$ were recruited. Subjects were excluded if they had significant current or previous medical history, were receiving regular medication affecting the GI or nervous systems, consumed $>20$ units of alcohol/week, or were smokers. Written consent for the study was obtained from every subject after a full explanation of the purpose and nature of all procedures used. The investigation was approved by the University of Surrey Advisory Committee on Ethics, functioning according to the guidelines issued by the Royal College of Physicians of London in August 1996.

\section{Study protocol}

The study design was a within-subject randomised crossover comparison of hormone concentrations in plasma and saliva following a mixed meal which was either chewed and swallowed, or chewed and expectorated (sham-fed).

The mixed test meal (total energy content $1708 \mathrm{~kJ}$, containing $40 \%$ total energy as carbohydrate, $40 \%$ as fat and $25 \%$ as protein) consisted of cream cheese and crackers, together with a $200 \mathrm{ml}$ glass of sugar-free orange squash. On each test occasion, after an overnight fast, a cannula was inserted into the subject's antecubital vein and two basal blood samples were taken. At the same time, two saliva samples were also taken. Subjects were then given the test meal. On one occasion the meal was chewed and swallowed (the swallowed meal), on the other it was chewed and expectorated (the sham-fed meal). Blood and saliva samples were simultaneously taken at intervals for $90 \mathrm{~min}$ following the meals (15, 30, 45, 60 and $90 \mathrm{~min})$. Blood and saliva samples were both centrifuged at $1825 \mathrm{~g}$ for $10 \mathrm{~min}$ to separate plasma from whole blood and any particulate matter from saliva. They were stored at $-20{ }^{\circ} \mathrm{C}$ until analysis.

\section{Assay procedures}

Plasma glucose and non-esterified fatty acid (NEFA) and salivary total protein and $\alpha$-amylase were analysed by standard automated enzymatic spectrophotometric methods (reagent kits from Roche Products Ltd, Welwyn Garden City, Herts, UK (glucose and protein), Wako Chemicals, Neuss, Germany (NEFA) and Randox, Country Antrim, Northern Ireland, UK ( $\alpha$-amylase)). The interassay coefficients of variation were less than $5 \%$ for these assays. Plasma and salivary insulin, C-peptide, GIP and GLP-1 were measured by in-house RIAs (Morgan et al. 1978, Elliott et al. 1993, Hampton \& Withey 1996). The interassay coefficients of variation were less than $10 \%$ for these assays. All samples from a single subject were measured in the same assay.

GIP and insulin-like immunoreactive species in saliva were considered to be similar to their gut and pancreatic counterparts on the grounds that (i) serially diluted saliva samples containing GIP and insulin ran parallel to standard curves constructed from GIP and insulin of GI origin, (ii) recovery of GIP and insulin in saliva samples 'spiked' with the hormones of GI origin was $104 \pm 5 \cdot 0$ and $106 \pm 6 \cdot 4 \%$ respectively, and (iii) GIP and insulin-like immunoreactivity in saliva samples co-eluted with their respective GI and pancreatic counterparts on HPLC (4.6 i.d. $\times 250 \mathrm{~mm}$ $5 \mu \mathrm{C} 18$ column; trifluoroacetic acid/acetonitrile solvent gradient) (Messenger 2000).

\section{Statistical analyses}

All hormone and metabolic data were compared by repeated-measures ANOVA with time and treatment as within-subject factors, followed by a post-hoc Duncan's 


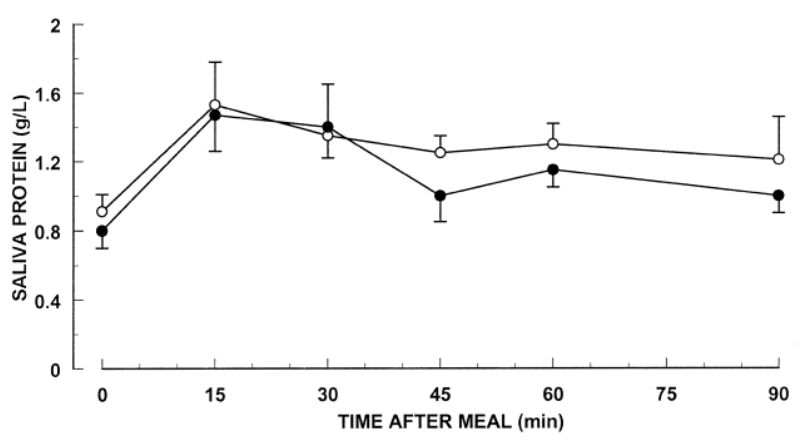

Figure 1 Salivary total protein levels in healthy subjects following a $1707 \mathrm{~kJ}$ test meal which was either swallowed $(\mathrm{O})$ or sham-fed (-). Means \pm S.E.M., $n=12$.

multiple range test of critical ranges, where appropriate, using the Statistica statistical package (Statsoft, Tulsa, OK, USA).

\section{Results}

\section{Postprandial metabolite responses}

Postprandial plasma levels of both glucose and NEFA were significantly different following the two test meals $(P<0 \cdot 01)$. Both were unchanged following the sham-fed meal. Mean glucose levels rose from basal levels of $4 \cdot 87 \pm 0 \cdot 1 \mathrm{mmol} / 1$ to a maximum of $5 \cdot 26 \pm 0 \cdot 2 \mathrm{mmol} / 1$ at $30 \mathrm{~min}$ and NEFA levels fell from basal levels of $0 \cdot 47 \pm 0 \cdot 04 \mathrm{mmol} / 1$ to plateau at $0 \cdot 18 \pm 0 \cdot 02 \mathrm{mmol} / \mathrm{l}$, 60 min following the swallowed meal.

Salivary total protein and $\alpha$-amylase concentrations following the two test meals are shown in Figs 1 and 2 . Salivary total protein rose following both sham-fed and swallowed meals, to reach peak levels at $30 \mathrm{~min}$. There were no significant differences between the sham-fed and swallowed meals $(P=0 \cdot 38)$. $\alpha$-Amylase activity also rose following both meals to reach peak levels at $15 \mathrm{~min}$ following the swallowed meal and $60 \mathrm{~min}$ following

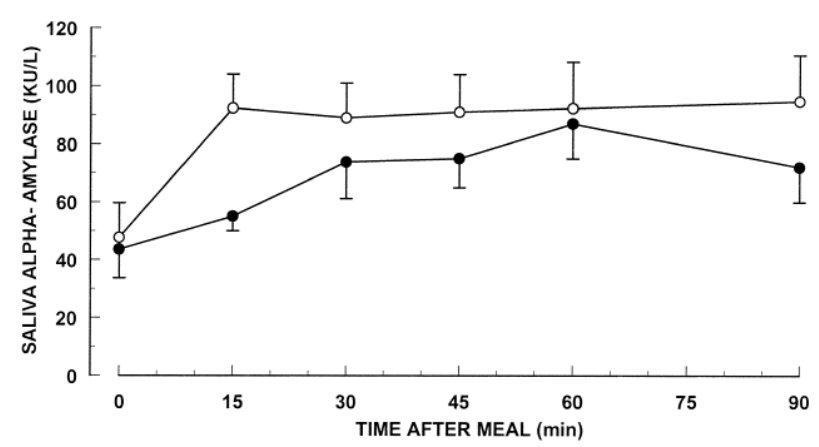

Figure 2 Salivary $\alpha$-amylase levels in healthy subjects following a $1707 \mathrm{~kJ}$ test meal which was either swallowed $(O)$ or sham-fed (-). Means \pm S.E.M., $n=12$.

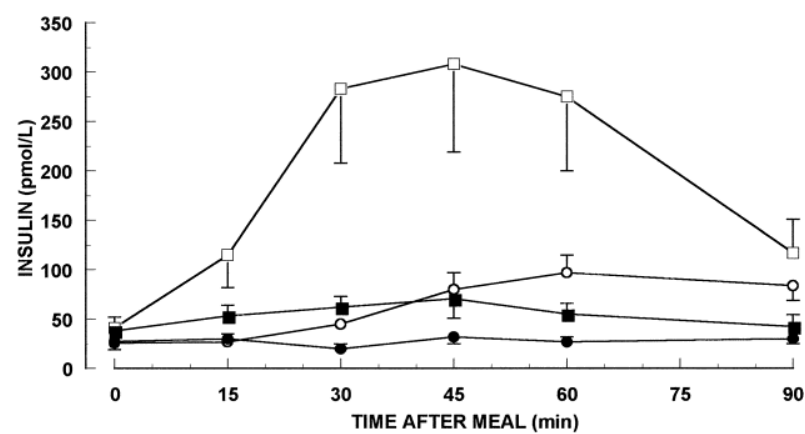

Figure 3 Salivary and plasma insulin levels in healthy subjects following a $1707 \mathrm{~kJ}$ test meal which was either swallowed or sham-fed. Means \pm S.E.M., $n=12$. $\bigcirc=$ saliva, swallowed meal;

- = saliva, sham-fed meal; $\square=$ plasma, swallowed meal;

- = plasma, sham-fed meal.

the sham-fed meal. The total response was significantly higher following the swallowed than the sham-fed meal $(P<0 \cdot 05)$.

\section{Postprandial hormone responses}

Plasma and salivary insulin levels following the two test meals are shown in Fig. 3. Plasma levels were significantly different following the two meals $(P<0 \cdot 01)$, remaining unchanged following the sham-fed meal but rising significantly $15 \mathrm{~min}$ following the swallowed meal, to reach a peak at $45 \mathrm{~min}$. Salivary insulin levels were also significantly different following the two meals $(P<0 \cdot 01)$, remaining unchanged following the sham-fed meal but rising more slowly than plasma levels, to reach a peak $60 \mathrm{~min}$ following the swallowed meal. Fasting salivary insulin concentrations were similar to those in plasma; however, the insulin response to a swallowed meal was significantly lower in saliva than in plasma $(P<0 \cdot 01)$.

Plasma and salivary GIP levels following the two test meals are shown in Fig. 4. Plasma levels were significantly

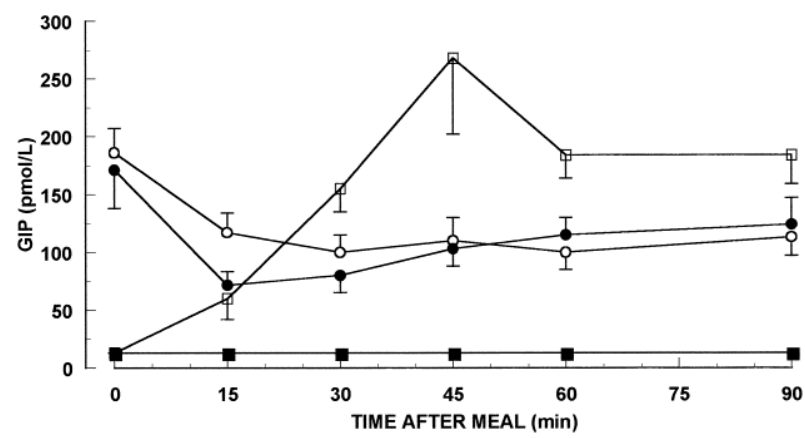

Figure 4 Salivary and plasma GIP levels in healthy subjects following a $1707 \mathrm{~kJ}$ test meal which was either swallowed or sham-fed. Means \pm S.E.M., $n=12$. $\bigcirc=$ saliva, swallowed meal; $\boldsymbol{0}$ = saliva, sham-fed meal; $\square=$ plasma, swallowed meal;

= plasma, sham-fed meal. 
different following the two meals $(P<0 \cdot 01)$, remaining unchanged following the sham-fed meal but rising significantly following the swallowed meal, to reach a peak at $45 \mathrm{~min}$. Fasting salivary GIP levels were significantly higher than the following plasma levels. They decreased immediately following both sham-fed and swallowed meals and remained significantly lower than fasting values for the rest of the study $(P<0 \cdot 025)$.

Plasma GLP-1 levels following the two test meals followed a similar pattern to plasma GIP. Basal circulating GLP-1 levels were $8 \cdot 6 \pm 2 \cdot 2 \mathrm{pmol} / 1$, rising to mean peak values of $29 \pm 4 \cdot 2 \mathrm{pmol} / 130 \mathrm{~min}$ following the swallowed meal. Plasma levels were unaffected by the sham-fed meal. No GLP-1-like immunoreactivity could be detected in fasting saliva samples, or following either meal.

\section{Discussion}

Plasma levels of hormones and metabolites were measured in order to enable a direct comparison between circulating and salivary hormones concentrations, to elucidate the origin of hormones found in saliva, and to verify that none of the food in the sham-fed meal was inadvertently swallowed. Plasma insulin and glucose levels rose and plasma NEFA fell in a manner typical of data that we have previously obtained following ingestion of a mixed meal and its absorption from the gut (Elliott et al. 1993, Lund et al. 2001). The lack of any change in plasma hormone or metabolite levels following the sham-fed meal is consistent with none of the meal contents being swallowed.

In contrast to plasma hormone and metabolite profiles, which were unchanged following the sham-fed meal, but which were altered in response to the swallowed meal, salivary total protein increased following both sham-fed and swallowed meals and the responses in saliva were similar for both meals. This would indicate that the signal for salivary protein release comes from the buccal cavity. The concentration of total protein in saliva has not previously been reported in response to food, but the stimulation of the salivary flow by the presence of food in the mouth has been widely investigated (Turner \& Camden 1990, Turner 1993). Saliva contains many biologically active compounds, including the enzyme $\alpha$-amylase, which is secreted from serous cells within the parotid gland, a gland which contributes approximately $20 \%$ of the total unstimulated saliva volume. Although $\alpha$-amylase activity rose following both meals, indicating that a signal for its release originates in the buccal cavity, the response following the sham-fed meal was significantly blunted and delayed, suggesting that a gastric, or postabsorptive stimulus occurs for secretion of $\alpha$-amylase into saliva, in addition to the oral one.

Insulin-like immunoreactivity in saliva increased following the swallowed meal but remained unchanged following the sham-fed meal. Salivary insulin levels in this study are consistent with those previously reported in the literature following a meal (Fekete et al. 1993). The pattern in saliva was very similar to that for plasma insulin. However, insulin levels in saliva rose more slowly and were lower than their corresponding plasma concentrations. The origin of immunoreactive insulin in saliva is of interest. Both immunoreactive insulin and insulin mRNA have been found in the salivary glands of mice (Kerr et al. 1995) and rats (Taouis et al. 1995), and immunoreactive insulin secretion from mouse salivary glands is sensitive to changes in glucose concentrations (Shubnikova et al. 1984). It is therefore possible that the insulin found in saliva could be the product of local synthesis. However, the correspondence between circulating and salivary insulin concentrations together with lower insulin levels in saliva makes it more likely that salivary insulin represents an ultrafiltrate from blood. The observation that salivary insulin has been shown to increase following an i.v. injection of insulin in humans, dissociated from any rise in blood glucose concentrations (Vallejo et al. 1984), supports this hypothesis.

No immunoreactive GLP-1 was detected in saliva. However, early reports of glucagon-like activity in salivary glands could be accounted for either by the non-specificity of the antiserum used, or by an artefact of salivary protease activity (Tahara et al. 1983).

To the best of our knowledge, this study is the first to report the presence of immunoreactive GIP in human saliva. GIP mRNA has only been detected within the GI tract and the salivary glands (Tseng et al. 1993). The GIP found in saliva must therefore originate from one of these sources. Peptide endocrine hormones are able to enter the saliva from the circulation via the ultrafiltration route, through the tight junctions between the salivary acinar cells. However, their concentration in saliva is typically several-fold lower than the corresponding concentrations in blood and saliva levels mimic blood levels (Vining et al. 1983). This was what was observed in our study for insulin, leading to our conclusion that insulin in saliva is probably an ultrafiltrate of blood. In contrast, however, immunoreactive GIP in saliva was some 9-fold higher than the corresponding plasma level in the fasting state and the pattern of GIP secretion in saliva following the meals bore no resemblance to the corresponding plasma profiles. It is therefore very unlikely that salivary GIP is an ultrafiltrate of blood (originating from GIP cells in the GI tract), but is instead much more likely to be a product of local synthesis. The demonstration of parallelism, good recovery and co-elution on HPLC with GIP of GI origin indicates that the molecular species in saliva identified by immunoassay is similar to the peptide found in the GI tract. The putative physiological role for GIP in saliva is of interest. A major function of GIP of GI origin is the potentiation of glucose-induced insulin secretion via the entero-insular axis (Morgan 1996), but the fall in salivary GIP following both meals mediates against GIP in saliva playing any part 
in stimulating postprandial insulin secretion. However, GIP was initially isolated and characterised on the basis of its gastric acid inhibitory properties in denervated stomach pouches of dogs (Brown et al. 1975), a biological activity which gave rise to its original name of 'gastric inhibitory polypeptide'. Interest in this biological activity waned when it was questioned whether circulating GIP levels were sufficiently high to inhibit gastric acid secretion from the intact human stomach, but interest in the enterogastrone activity of GIP has recently been revived (Rossowski et al. 2002). It is possible that GIP in saliva is the product of local salivary gland synthesis. Its secretion could be stimulated either directly, by oral stimuli, or regulated by central nervous stimuli, due perhaps to the anticipation of food ingestion. GIP is a low molecular mass peptide of 4000 Da, whose activity is preserved in an acid environment. Sham-feeding has been shown to be a potent stimulator of gastric acid secretion (Feldman \& Richardson 1986). The decrease in salivary GIP levels observed in this study following sham-feeding could enable gastric acid secretion to be released from an inhibitory control exerted by high levels of salivary GIP in the fasting state. However, in the absence of any additional evidence to support the descriptive findings of this study, any physiological role for salivary GIP must remain speculative. The biological activities of saliva have received comparatively little attention, and most work has concentrated on its local action within the buccal cavity. This study raises the intriguing possibility that GIP, transported in saliva rather than blood, might, in a manner analogous to its endocrine role, have biological actions at a site remote from its site of synthesis.

\section{Acknowledgement}

This work was funded by EU Project FAIR CT-95-0653.

\section{References}

Boyer R, Jame F \& Arancibia S 1991 A non-exocrine function of the submandibular salivary-gland. Annales d'Endocrinologie 52 307-322.

Brown JC, Dryburgh JR, Ross SA \& Dupre J 1975 Identification and actions of gastric inhibitory polypeptide. Recent Progress in Hormone Research 31 487-532.

Elliott RM, Morgan LM, Tredger JA, Deacon S, Wright J \& Marks V 1993 Glucagon-like peptide-1 (7-36)amide and glucose-dependent insulinotropic polypeptide secretion in response to nutrient ingestion in man: acute post-prandial and 24-h secretion patterns. Journal of Endocrinology 138 159-166.

Fekete Z, Korec R, Feketeova E, Murty VLN, Piotrowski J, Slomiany A \& Slomiany BL 1993 Salivary and plasma insulin levels in man. Biochemistry and Molecular Biology International 30 623-629.

Feldman M \& Richardson CT 1986 Role of thought, sight, smell and taste of food in the cephalic phase response of gastric acid secretion in humans. Gastroenterology 90 428-433.

Hampton SM \& Withey L 1996 Monitoring B-cell responses in obese and normal weight subjects: a pilot study. Diabetes and Metabolism $19582-585$.
Humphreysbeher MG, Macauley SP, Chegini N, Vansetten G, Purushotham K, Stewart C, Wheeler TT \& Schultz GS 1994 Characterization of the synthesis and secretion of transforming growth-factor-alpha from salivary-glands and saliva. Endocrinology 134 963-970.

Kato T, Hanaoka K, Ochiai O, Chikuma T, Takeda K, Yajima R \& Kumegawa M 1992 Secretion of peptidylglycine alpha-amidating monooxygenase (PAM) from rat salivary glands. Neurochemistry International 21 153-158.

Kerr M, Lee A, Wang PL, Purushotham KR, Chegini N, Yamamoto H \& Humphreysbeher MG 1995 Detection of insulin and insulin-like growth-factor-I and growth-factor-II in saliva and potential synthesis in the salivary-glands of mice - effects of type-1 diabetes mellitus. Biochemical Pharmacology 49 1521-1531.

Kimura I, Sasamura T, Tsuneki H, Iwai M, Furuta I, Sasamoto H \& Furusawa Y 1998 Immunochemical localization of salivary peptide $\mathrm{P}-\mathrm{C}$ in human submandibular gland. Biological and Pharmaceutical Bulletin 21 506-512.

Kimura M, Nakashima N \& Kimura I 1990 Salivary peptide P-c modulates both insulin and glucagon release from isolated perfused rat pancreas. Japanese Journal of Pharmacology 52 579-585.

Kimura M, Nakashima N \& Kimura I 1995 The essential amino acid domains in salivary peptide P-C that potentiate glucose-induced insulin release and inhibit arginine-induced glucagon release from perfused rat pancreas. Japanese Journal of Pharmacology 67 79-82.

Lawrence AM, Tan S, Hojvat S \& Kirsteins L 1977 Salivary gland hyperglycemic factor: an extrapancreatic source of glucagon like material. Science 195 70-72.

Lund J, Arendt J, Hampton SM, English J \& Morgan LM 2001 Postprandial hormone and metabolic responses amongst shift workers in Antarctica. Journal of Endocrinology 171 557-564.

Mattes RD 1996 Oral fat exposure alters postprandial lipid metabolism in humans. American Journal of Clinical Nutrition 63 911-917.

Messenger B 2000 Salivary gland peptide hormones and dietary phenols. PhD Thesis. University of Surrey.

Morgan LM 1996 The metabolic role of GIP: physiology and pathology. Biochemical Society Transactions 24 585-591.

Morgan LM, Morris BA \& Marks V 1978 Radioimmunoassay of gastric inhibitory peptide. Annals of Clinical Biochemistry 261 72-77.

Murakami K, Taniguchi H \& Baba S 1982 Presence of insulin-like immunoreactivity and its biosynthesis in rat and human parotid gland. Diabetologia 22 358-361.

Rossowski WJ, Cheng BL, Jiang NY \& Coy DH 2002 Examination of somatostatin involvement in the inhibitory action of GIP, GLP-1, amylin and adrenomedullin on gastric acid release using a new SRIF antagonist analogue. British Journal of Pharmacology 125 1081-1087.

Shubnikova EA, Volkova EF \& Printseva OY 1984 Submandibular glands as organs of synthesis and accumulation of insulin-like protein. Acta Histochemica 74 157-171.

Tahara Y, Shima K, Hirota M, Ikegami H, Tanaka A \& Kumahara Y 1983 Salivary gland glucagon is a fictitious substance due to tracer-degrading activity resistant to protease inhibitors. Biochemical and Biophysical Research Communications 113 340-347.

Taouis M, Deville de Perrier D, Hillaire-Buys D, Derouet M, Gross R, Simon J \& Ribes G 1995 Biological activity of immunoreactive insulin-like activity extracted from rat submandibular gland. American Journal of Physiology 269 E277-E282.

Teff KL \& Engleman K 1996 Oral sensory stimulation improves glucose tolerance in humans: effects on insulin, C-peptide and glucagon. American Journal of Physiology 270 R1371-R1379.

Tseng CC, Jarboe LA, Landau SB, Williams EK \& Wolfe MM 1993 Glucose-dependent insulintropic peptide: structure of the precursor and tissue specific expression in rat. PNAS 90 1992-1996.

Tseng CC, Jarboe LA \& Wolfe MM 1994 Regulation of glucose-dependent insulinotropic peptide gene expression by a glucose meal. American Journal of Physiology 266 G887-G891. 
Tseng CC, Boylan MO, Jarboe LA, Williams EK, Sunday ME \& Wolfe MM 1995 Glucose-dependent insulinotropic peptide (GIP) gene expression in the rat salivary gland. Molecular and Cellular Endocrinology 115 13-19.

Turner RJ 1993 Mechanisms of fluid secretion by salivary glands. Annals of the New York Academy of Sciences 694 24-35.

Turner JT \& Camden JM 1990 The influence of vasoactive intestinal peptide receptors in dispersed acini from rat submandibular salivary gland on cyclic AMP production and mucin release. Archives of Oral Biology 35 103-108.
Vallejo G, Mead PM, Gaynor DH, Devlin JT \& Robbins DC 1984 Characterisation of immunoreactive insulin in human saliva: evidence against production in-situ. Diabetologia 27 437-440.

Vining RF, McGinley RA \& Symons RG 1983 Hormones in saliva: mode of entry and consequent implications for clinical interpretation. Clinical Chemistry 29 1752-1756.

Received in final form 11 February 2003 Accepted 14 February 2003 